\title{
Estimación del incremento de las emisiones de contaminantes a la atmosfera producto del proceso de cremación de cadáveres por covid-19 en la ciudad de México
}

\author{
Sugey Aramis Benavides Fajardo \\ sabf.0496@gmail.com \\ Instituto Politécnico Nacional-UPIBI \\ CDMX - México \\ Nayeli Mariana Soto Cabrera \\ nayelisotocabrera7@gmail.com \\ Instituto Politécnico Nacional-UPIBI
}

CDMX - México

Lorena Ugalde Cerón lorenauga@hotmail.com

Instituto Politécnico Nacional-UPIBI

CDMX - México

Saúl Hernández Islas saulhernandezislas@yahoo.com.mx Instituto Politécnico Nacional-UPIBI

CDMX - México

Francisco Márquez Montesino marquezmontesino1992@gmail.com Departamento de Química Universidad de Pinar del Río "Hermanos Saíz Montes de Oca” Pinar del Río-Cuba

\section{RESUMEN}

En febrero del 2020 con la presencia del virus causante de la enfermedad COVID-19 en México, se incrementaron las cremaciones de personas fallecidas. Por tal razón se realizó la investigación sobre la cremación de cadáveres, cuya problemática fue identificar si la calidad del aire se vió afectada debido a estas cremaciones en la Ciudad de México, considerando si hay necesidad de corregir o cambiar la normatividad vigente, incluyendo la definición de cremación. Se determinó que hubo un incremento considerable de los contaminantes producto de las cremaciones en los años 2020 y 2021 respecto al 2019, detectándose la presencia de contaminantes que no son contemplados en la norma actual NADF-017-AIRE-2017. Estos contaminantes representan un alto riesgo de impacto a la salud de la población y el ambiente. Alrededor de 1,542,037.790 kg de ácido clorhídrico, 
aunado a $1,417.830 \mathrm{~kg}$ de plomo, con más de 70,462.560 kg de mercurio y $0.793 \mathrm{mg}$ de dioxinas y furanos, tuvieron un incremento al ser emitidos a la atmósfera de la CDMX. Por lo que se sugiere la actualización inmediata de la normatividad, incluyendo más Límites Máximos Permisibles de contaminantes producto de la cremación de cadáveres, como lo hacen Colombia, USA y parte de la Comunidad Europea.

Palabras claves: contaminación; atmósfera; cremación; incineración; pandemia. 


\title{
Estimation of the increase in pollutant emissions \\ to the atmosphere product of the cremation process of corpses \\ by covid-19 in Mexico City
}

\begin{abstract}
In February 2020, with the presence of the virus that causes the COVID-19 disease in Mexico, the cremations of deceased persons increased considerably. For this reason, the investigation was carried out on the cremation of corpses, whose problem was to identify if the air quality was affected due to these cremations in Mexico City, considering if there is a need to correct or change the current regulations, including the definition cremation. It was determined that there was a considerable increase in contaminants resulting from cremations in 2020 and 2021 compared to 2019, detecting the presence of contaminants that are not contemplated in the current standard NADF-017-AIRE-2017. These pollutants represent a high risk of impact on the health of the population and the environment. Around 1,542,037,790 kg of hydrochloric acid, together with 1,417,830 kg of lead, with more than 70,462,560 kg of mercury and $0.793 \mathrm{mg}$ of dioxins and furans, had an increase when emitted into the CDMX atmosphere. Therefore, the immediate update of the regulations is suggested, including more Maximum Permissible Limits of contaminants resulting from the cremation of corpses, as Colombia, USA and part of the European Community do.
\end{abstract}

Keywords: contamination; atmosphere; cremation; incineration; pandemic.

Artículo recibido: 05 octubre. 2021 Aceptado para publicación: 02 noviembre 2021

Correspondencia: sabf.0496@gmail.com Conflictos de Interés: Ninguna que declarar 


\section{INTRODUCCIÓN}

La Organización Panamericana de Salud (OPS) dice que en el Continente Americano mueren más de 131,000 personas en países de bajos ingresos y 96,000 en países de altos ingresos por causas vinculadas a la contaminación del aire ${ }^{(4)}$.

La Organización Mundial de la Salud (OMS) informa que, en el año 2012 unos siete millones de personas murieron ${ }^{(4)}$, esto quiere decir que una de cada ocho del total de muertes en el mundo, es consecuencia de la exposición a la contaminación atmosférica.

Actualmente en México, el proceso de cremación trae consigo diferentes efectos negativos, como lo menciona el Consejo Superior de Investigaciones Científicas. Este menciona que un cuerpo humano emite aproximadamente $27 \mathrm{~kg}$ de Dióxido de Carbono cuando es quemado ${ }^{(5)}$; lo que se convierte en un problema directo para la zona donde se programa colocar un crematorio o donde se ubica, ya que los contaminantes emitidos pueden representar efectos negativos para las comunidades aledañas a dichos crematorios.

"Cremar en los centros urbanos presenta inconvenientes a corto y largo plazo para las comunidades aledañas. A las concentraciones de óxidos de carbono, azufre y nitrógeno, sumando al material particulado $\left(\mathrm{PM}_{10}\right.$ y $\left.\mathrm{PM}_{2,5}\right)$ e hidrocarburos poli aromáticos, como menciona la norma en la resolución 2257 de 2017, también se agregan reportes de emisión de metales pesados como mercurio, plomo y cadmio". Estas son las palabras utilizadas por el químico y experto en toxicología, Fredy Vergara ${ }^{(6)}$. Las instalaciones de incineración han de diseñarse, equiparse, construirse y explotarse de modo que en los gases de escape no se superen los valores límite de emisión establecidos, por lo que se necesita esté legislado para un gran número de contaminantes: partículas totales, $\mathrm{CO}$, compuestos orgánicos totales (COT), $\mathrm{HCl}, \mathrm{HF}, \mathrm{SO}_{2}$, óxidos de nitrógeno ( $\mathrm{NOx}$ ), metales pesados ( $\mathrm{Hg}, \mathrm{Cd}, \mathrm{Tl}, \mathrm{Sb}, \mathrm{As}, \mathrm{Pb}, \mathrm{Cr}, \mathrm{Co}, \mathrm{Cu}, \mathrm{Mn}, \mathrm{Ni} \mathrm{y} \mathrm{V})$, dioxinas y furanos (PCDD/PCDF).

Así mismo un estudio sobre dichas emisiones publicado en México en la Gaceta del Senado el 02 de octubre del $2018^{(3)}$, presentó que, en la cremación de cadáveres, se producen contaminantes que no están incluidos en la normatividad existente y de forma evidente no son monitoreados.

La problemática principal incide a partir de que llegó el COVID-19 a México, el primer caso identificado se presentó el 27 de febrero de 2020, con ello el número de pacientes 
aumentó exponencialmente alcanzando un total de 19,224 casos confirmados y de esos 1,859 (9.67\%) fallecidos a sólo 64 días después del primer diagnóstico ${ }^{(7)}$. Considerando el inicio de la pandemia hasta el 1 de julio del 2021, en México se acumuló un total de 2,525,350 casos confirmados y un total de 233,248 fallecidos por COVID-19. En la Ciudad de México desde el inicio de la pandemia y hasta el 1 de julio de 2021, se registraron un total de 686,955 casos confirmados, mientras que las personas fallecidas por COVID-19 sumaron 34,708 ${ }^{(8)}$. Debido a la cantidad de decesos en el mundo y al impacto que tuvo dicho suceso, se modificó el proceso de disposición final de estos cadáveres, como menciona la Secretaría de Salud en la Guía de Manejo de Cadáveres por COVID-19 (SARS-CoV-2) en México: “La disposición final del cadáver deberá ser lo más pronto posible, preferiblemente mediante la cremación; y de no ser posible, se llevará a cabo la inhumación en sepultura o bóveda. De ser por entierro, este se dará en condiciones habituales. Las cenizas pueden ser objeto de manipulación sin que supongan ningún riesgo" ${ }^{(9)}$.

Debido a la cantidad de cremaciones, la sociedad ha resentido el desarrollo excesivo de dicho proceso en sus vidas habituales, presentando afectaciones en su salud y en su estilo de vida. Crematorios como el de San Isidro, localizado en Azcapotzalco (CDMX), presentaron casos como el siguiente: la auditora ambiental acreditada por la EMA y aprobada por la PROFEPA en los rubros de aire, ruido ambiental, riesgo ambiental, residuos, suelo, subsuelo y agua, Griselda González Cardoso, indicó que debido a la frecuencia del proceso de cremación no es posible llevar a cabo una buena combustión; de igual forma presentó una sugerencia a los vecinos de la colonia San Pedro Xalpa, en la que les recomendaba que presentasen una solicitud de monitoreo de partículas $\mathrm{PM}_{10} \mathrm{y}$ $\mathrm{PM}_{2.5}$ a la Secretaría del Medio Ambiente de la Ciudad de México (SEDEMA) y a la Secretaría del Medio Ambiente y Recursos Naturales (SEMARNAT), para que tuvieran conocimiento de dichas emisiones. "Las emisiones de partículas son de 40 miligramos por metro cúbico, son los Límites Máximos Permisibles, los hornos crematorios tienen que realizar estas mediciones una vez al año para que puedan reportarlos en la Licencia Ambiental Única de la Ciudad de México, sin embargo, por esta emergencia se podría monitorear para saber si estamos rebasando estos LMP que establece la norma" (10).

Por todo lo anterior se realizó la investigación sobre la cremación de cadáveres por COVID-19, en donde la problemática es que durante la pandemia la calidad del aire de 
México y específicamente de la CDMX no mejoró, ni ha existido una variación positiva a diferencia de muchos otros países. Por tal razón se propuso como objetivo general determinar el tipo y la cantidad de emisiones a la atmósfera producto del incremento de la cremación de cadáveres por COVID-19 en la Ciudad de México, en los periodos enero 2020 a junio 2021 en comparación con el año 2019.

Así, el presente trabajo muestra los cambios en los datos de contaminantes atmosféricos, al comparar las emisiones antes y las estimadas durante dicha pandemia, es decir, en los años 2019, 2020 y el primer semestre del 2021, específicamente en la CDMX; demostrándose que existen huecos normativos e incógnitas entre las diferentes normatividades de competencia atmosférica para fuentes fijas.

Otra problemática identificada fue la definición que se utiliza entre las palabras cremación e incineración, puesto que se llegan a usar de forma igual o indistinta, por ejemplo en los diccionarios o en internet al buscar la palabra cremación, automáticamente se define como la acción de incinerar, a veces incluso sin definir qué es lo que se incinera, creando confusión o distorsión, problemática reflejada también en la NADF-017-AIRE-2017 ${ }^{(1)}$, puesto que el término incineración se define como: "Proceso mediante el cual los cadáveres de animales y sus restos se someten a altas temperaturas controladas con objeto de reducirlos a cenizas", y en esa misma norma la cremación se define como: "Proceso mediante el cual un cadáver, restos humanos o restos humanos áridos se someten a altas temperaturas controladas con el objeto de reducirlos a cenizas" (1). Claramente podemos observar que lo único que cambia en estas definiciones es que en una se incineran animales o restos, y en la otra cadáveres.

Mientras tanto en la NOM-098-SEMARNAT-2002, la definición de incineración es: “Cualquier proceso para reducir el volumen y descomponer o cambiar la composición física, química o biológica de un resto sólido, liquido o gaseoso, mediante oxidación térmica, en la cual todos los factores de combustión como la temperatura, el tiempo de retención y la turbulencia, pueden ser controlados, a fin de alcanzar la eficiencia y los parámetros ambientales previamente establecidos." (2)

Por todo lo anterior se planteó que para cadáveres, se maneje el término cremación de forma independiente a incineración y se tenga una definición propia, también qué al existir deficiencias en la normatividad aplicable a las cremaciones, merece haber actualizaciones urgentes a las mediciones de muchos más contaminantes atmosféricos, 
para así concluir y profundizar mediante los conocimientos teóricos sobre posibles efectos en el ambiente y en la salud humana, ayudando a la concientización de la población local.

\section{ESTRATEGIAS METODOLÓGICAS O MATERIALES Y MÉTODOS}

El presente trabajo se llevó a cabo mediante la aplicación de una investigación documental. Es importante mencionar que debido a la pandemia por COVID-19, solo se pudo solicitar información a las autoridades correspondientes a través de correo electrónico y en las páginas oficiales; resaltando la escasa respuesta a la información solicitada.

Las autoridades o dependencias a las cuales puntualmente se les solicitó información, fueron las siguientes:

- Secretaría del Medio Ambiente y Recursos Naturales

- Jefatura de Gobierno de la Ciudad de México

- Dirección de Cultura Ambiental de la Dirección General de Coordinación de Políticas y Educación Ambiental de la Secretaría de Medio Ambiente

- Secretaría del Medio Ambiente de la Ciudad de México

- Secretaria de Salud de la Ciudad de México

- Dirección General de la Calidad del Aire

\section{Metodología}

2.1. Fundamentación teórica documental de la cremación en el mundo y en México.

2.1.1. Identificación de los factores de emisión de contaminantes a la atmosfera emitidos en el proceso de la cremación de un cadáver, factores que actualmente se utilizan en la USEPA.

2.2. Recopilación de antecedentes históricos de la cremación.

2.3. Establecimiento de Objetivos.

2.4. Establecimiento de definiciones de acuerdo con las normas NADF-017-AIRE-2017 y NOM-098-SEMARNAT-2002.

2.5. Investigación del proceso y emisiones antes de la pandemia:

2.5.1. Se realizó la investigación conforme al proceso normativo y teórico, encontrado en diferentes fuentes. 
2.6. Recopilación de información sobre los contaminantes producidos al cremar un cadáver

2.7. Cuantificación (estimación) de emisiones producidas antes, durante y después de la pandemia.

2.7.1. Con los datos ya establecidos con anterioridad, se realizó la cuantificación de las emisiones producidas por los cadáveres cremados en la CDMX acorde a las normas establecidas:

2.7.1.1. Norma NADF-017- AIRE-2017(1) - Equipos de cremación e incineraciónLímites Máximos Permisibles de emisiones a la atmósfera y condiciones de operación.

Para la instalación y la operación, deberán contar con los permisos y autorizaciones previamente establecidas en el marco jurídico aplicable, y contar con la Licencia Ambiental Única para Ciudad de México (antes Distrito Federal).

Tabla 1. Límites Máximos Permisibles (Frecuencia de medición: 1 vez al año) ${ }^{(1)}$

\begin{tabular}{|cc|}
\hline $\begin{array}{c}\text { Contaminante } \\
\text { unidades }\end{array}$ & $\begin{array}{c}\text { Límite máximo de emisión } \\
\text { promedio horario } \\
(\mathbf{m g} / \mathbf{m} 3)\end{array}$ \\
\hline Partículas Suspendidas & 40 \\
\hline Monóxido de Carbono $(\mathrm{CO})$ & 120 \\
\hline Óxidos de Nitrógeno $(\mathrm{NOx})$ & 180 \\
\hline
\end{tabular}

*Nota: El cumplimiento de los LMP se debe determinar mediante la medición directa en cada fuente y chimenea, (condiciones de $25{ }^{\circ} \mathrm{C}, 1$ Atmósfera y $11 \%$ de Oxígeno).

Las mediciones para comprobar el cumplimiento de la Norma deberán realizarse por un laboratorio aprobado y registrado ante el Padrón de Laboratorios Ambientales reconocido por el Gobierno de la Ciudad de México.

2.7.1.2 NOM-098-SEMARNAT-2002 ${ }^{(2)}$-Establecimiento de las especificaciones de operación, así como los Límites Máximos Permisibles de emisión de contaminantes a la atmósfera para las instalaciones de emisión de residuos, considerada por los contaminantes que maneja la norma.

En la tabla 2 se encuentran los límites máximos permisibles (LMP) de emisiones para las instalaciones de incineración de residuos y la frecuencia con la que estos deben ser medidos. 
Tabla 2. Límites Máximos Permisibles de emisiones para las instalaciones de incineración de residuos y la frecuencia. ${ }^{(2)}$

\begin{tabular}{|c|c|c|}
\hline Contaminante & Límites de emisión & $\begin{array}{l}\text { Frecuencia de } \\
\text { medición }\end{array}$ \\
\hline $\mathrm{CO}\left[\mathrm{mg} / \mathrm{m}^{\wedge} 3\right]$ & 63 & Continuo \\
\hline $\mathrm{HCl}\left[\mathrm{mg} / \mathrm{m}^{\wedge} 3\right]$ & 15 & Trimestral \\
\hline $\mathrm{NOx}\left[\mathrm{mg} / \mathrm{m}^{\wedge} 3\right]$ & 300 & Semestral \\
\hline $\mathrm{SO}_{2}\left[\mathrm{mg} / \mathrm{m}^{\wedge} 3\right]$ & 80 & Semestral \\
\hline Partículas suspendidas $\left[\mathrm{mg} / \mathrm{m}^{\wedge} 3\right]$ & 50 & Semestral \\
\hline $\begin{array}{c}\text { Arsénico, Selenio, Cobalto, Níquel, } \\
\text { Magnesio, Estaño } \\
{\left[\mathrm{mg} / \mathrm{m}^{\wedge} 3\right]}\end{array}$ & $0.7 *$ & Semestral \\
\hline Cadmio $\left[\mathrm{mg} / \mathrm{m}^{\wedge} 3\right]$ & 0.07 & Semestral \\
\hline $\begin{array}{l}\text { Plomo, Cromo total, Cobre, Zinc } \\
\qquad\left[\mathrm{mg} / \mathrm{m}^{\wedge} 3\right]\end{array}$ & $0.7 *$ & Semestral \\
\hline Mercurio $\left[\mathrm{mg} / \mathrm{m}^{\wedge} 3\right]$ & 0.07 & Semestral \\
\hline $\begin{array}{l}\text { Dioxinas y furanos EQT (instalaciones } \\
\text { nuevas) } \\
{\left[\mathrm{ng} / \mathrm{m}^{\wedge} 3\right]}\end{array}$ & 0.2 & Anual \\
\hline $\begin{array}{l}\text { Dioxinas y furanos EQT (instalaciones } \\
\text { existentes antes de la norma) } \\
\qquad\left[\mathrm{ng} / \mathrm{m}^{\wedge} 3\right]\end{array}$ & 0.5 & Anual \\
\hline
\end{tabular}

*suma total de metales pesados

2.7.1.3. Guía de manejo de cadáveres por COVID-19 SARS-COV-2 en México. (abril 5 del 2020).

\section{RESULTADOS Y DISCUSIÓN}

\subsection{Emisiones a la atmósfera producto de la cremación}

Antes de iniciar con el cálculo (estimación) de emisiones a la atmósfera producto de la cremación de cadáveres por COVID-19, se consideraron los contaminantes y los factores de emisión (producto de la cremación de cadáveres) proporcionados por la Agencia de Protección Ambiental de Estados Unidos y de la Guía de inventario de emisiones de contaminantes atmosféricos, del Programa Europeo de Seguimiento y Evaluación/ Espacio Económico Europeo (EMEP/EEA), información que se muestra en la tabla 3 $(11,12)$. 
Tabla 3. Factores de emisión en la cremación, de la USEPA y EMEP/EEA, proporcionados por la página española "funeral natural: a sustainable death" (13).

\begin{tabular}{|c|c|c|}
\hline \multicolumn{3}{|c|}{ Factores de emisiones en la cremación } \\
\hline Pollutant & Emission Factor (mg/body) & Source \\
\hline $\mathrm{SO}_{2}$ & 0.000000544 & EMEP/EEA, 2009 \\
\hline $\mathrm{NO}_{2}$ & 0.000000349 & EMEP/EEA, 2009 \\
\hline $\mathrm{CO}$ & 0.000000141 & EMEP/EEA, 2009 \\
\hline $\mathrm{PM}^{2}$ & 38555.351 & USEPA, 1992 \\
\hline Lead & 30.028 & USEPA, 1992 \\
\hline Hydrogen Fluoride & 297.103 & USEPA, 1992 \\
\hline Arsenic and compounds & 13.608 & USEPA, 1992 \\
\hline Cadmium VI compounds & 5.035 & USEPA, 1992 \\
\hline Chromium and compounds & 6.123 & USEPA, 1992 \\
\hline Dioxins and Furans & 0.0000168 & USEPA, 1992 \\
\hline Antimony and compounds & 13.698 & USEPA, 1992 1992 \\
\hline Barium & 13.608 & USEPA, 1992 \\
\hline Copper fumes & 12.428 & USEPA, 1992 \\
\hline Hydrogen Chloride & 32658.651 & USEPA, 1992 \\
\hline Note 2: Total PM assumed to be 100\% PM 10 or PM 2.5 & 1492.319 & \\
\hline Los factores de emisión presentados en esta tabla incluyen la combustión del féretro con el \\
cuerpo y el combustible del horno empleado, propano para los factores de emisión USEPA y gas \\
natural para los de EMEP/EEE.
\end{tabular}

En segundo término, se estimó la cantidad de cadáveres que se cremaban antes de la pandemia (2019) y durante la pandemia (enero 2020 a junio 2021).

Respecto a la cantidad de personas fallecidas en 2019, se consideraron las defunciones registradas por entidad federativa según su tipo, en este caso de la CDMX. Dicha información fue obtenida de una nota técnica emitida por el Instituto Nacional de Estadística y Geografía (INEGI) en 2019 (tabla 1 del anexo, referencia 14) la cual indica 
número de defunciones registradas por entidad federativa en 2019 que fueron 77,353 muertes totales. ${ }^{(14)}$

Para obtener el \% de cremaciones en el año 2019, se consideró la información proporcionada por las autoridades correspondientes (SEDEMA), mediante la cual se obtuvo información a partir de la funeraria J. García López, donde se plantea el porcentaje de cremaciones en la CDMX, que fue del $65 \%$ para el año $2017^{(15)}$, mientras que en el documento proporcionado por la INEGI titulado "recopilación de información de los panteones públicos en la zona Metropolitana del país, 2020”(16), en el cual se mencionan las muertes y cremaciones por COVID-19 en todo México, y él \% de cremación para el año 2020 que incremento al 76.8\%. Con base al comportamiento de estos datos se realizó la siguiente interpolación:

$\%$ cremación $2019=65 \%+\frac{76.8 \%-65 \%}{2020-2017}(2019-2017)=72.9 \%$

Obteniendo así que alrededor de 72.9\% de las personas en 2019 fueron cremadas.

Una vez obtenido el número de muertes en 2019 y un estimado del porcentaje de cremaciones, se calculó que alrededor de 56,359 personas fueron cremadas, usando este dato para calcular el total de $\mathrm{mg} /$ cuerpo de los contaminantes analizados por la normatividad mexicana, información mostrada en la tabla 4.

Se muestra el siguiente cálculo respecto al contaminante PM para los años 2019, 2020 y 2021 (destacando que en 2021 solo se consideró el primer semestre que fue de enero a junio). Los cálculos fueron los mismos para todos los contaminantes.

Factor de emisión (2009) PM = 38,555.351 $\frac{\mathrm{mg}}{\text { cuerpo }}$

- Muertes del $2019=77,353$ personas

$\%$ Cremaciones $=72.9 \%$

- Muertes del $2020=132,751$ personas

$\%$ Cremaciones $=76.8 \%$

- Muertes del $2021=75,499$ personas

$\%$ Cremaciones $=76.8 \%$

Se multiplican la cantidad de personas por el \% de cremaciones para así poder obtener un estimado de la cantidad de personas que fueron cremadas.

- Cremaciones $=$ Muertes $* \%$ Cremación

- Cremaciones $2019=56,359$ cremaciones

- Cremaciones $2020=101,953$ cremaciones

- Cremaciones $2021=57,983$ cremaciones 
Posteriormente multiplicamos esas cremaciones por el factor de emisión para obtener los $\frac{m g}{\text { cuerpo }}$ de las personas cremadas que fueron emitidos al ambiente.

Contaminante PM $\left[\frac{m g}{\text { cuerpo }}\right]=$ Factor de emisión * Cremaciones

- En 2019 se produjeron - PM =2,172,956,287 $\frac{\mathrm{mg}}{\text { cuerpo }}$

- En 2020 se produjeron - PM =3,930,824,755.662 $\frac{\mathrm{mg}}{\text { cuerpo }}$

- En 2021 se produjeron - PM =2,235,549,056.620 $\frac{\mathrm{mg}}{\text { cuerpo }}$

En cuanto al cálculo de la variación de los contaminantes, se hizo una resta del contaminante emitido en 2020 y 2021 menos el contaminante emitido en 2019.

Variación de $2019-2020=3,930,824,755.662 \frac{\mathrm{mg}}{\text { cuerpo }}-2,172,956,287 \frac{\mathrm{mg}}{\text { cuerpo }}=1,757,868,468.445 \frac{\mathrm{mg}}{\text { cuerpo }}$

Pasando los $\frac{m g}{\text { cuerpo }}$ a kg de contaminante: $1,757,868.47 \mathrm{~kg}$ de contaminante

Variación de $2019-2021=2,235,549,056.620 \frac{\mathrm{mg}}{\text { cuerpo }}-2,172,956,287 \frac{\mathrm{mg}}{\text { cuerpo }}=62,592,769.403 \frac{\mathrm{mg}}{\text { cuerpo }}$

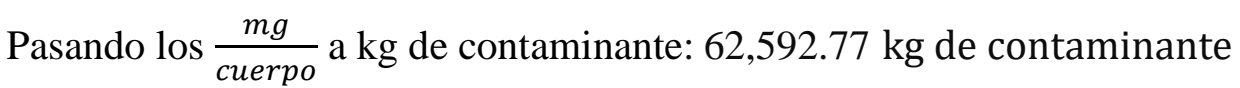

Para calcular el incremento total del contaminante PM, se llevó a cabo la suma de ambas variaciones 2019 - 2020 y $2019-2021$

Incremento total $=1,757,868.47 \mathrm{~kg}$ de contaminante $+62,592.77 \mathrm{~kg}$ de contaminante Incremento total $=1,820,461.24 \mathrm{~kg}$ del contaminante PM

Nota: El único valor que se deja en mg es el valor de las Dioxinas y Furanos, debido a que en la norma esta presentado con valores de EQT que refiere a $\left[\mathrm{mg} / \mathrm{m}^{3}\right]$ porque es toxico y peligroso a concentraciones muy bajas y por ende el valor se deja registrado en mg.

Tabla 4. Resultados de contaminantes totales en mg/cuerpo, que sí son medidos por la norma NADF-017-AIRE-2017 (del año 2019).

\begin{tabular}{|c|c|c|}
\hline Contaminante & Factor de emisión (mg/cuerpo) & $\begin{array}{c}\text { Total, por cuerpos en 2019 } \\
\text { (mg/cuerpo) }\end{array}$ \\
\hline Partículas Suspendidas & $38,555.35$ & $2,172,956,287$ \\
\hline Monóxido de Carbono & $1.41 \times 10^{-7}$ & $7.95 \times 10^{-03}$ \\
\hline Óxidos de nitrógeno & $3.09 \times 10^{-7}$ & $1.74 \times 10^{-02}$ \\
\hline
\end{tabular}


Respecto a la cantidad de personas fallecidas en 2020, se consideraron las defunciones registradas por entidad federativa de registro según sexo, en este caso de la CDMX. Dicha información fue obtenida de una nota técnica emitida por el Instituto Nacional de Estadística y Geografía (INEGI) en 2021 (tabla 1 de anexo 2, referencia 17) la cual indica número de defunciones en 2020 que fueron 132,751 muertes totales ${ }^{(17)}$.

Para obtener el \% cremación se obtuvo información de INEGI del documento público "recopilación de información de los panteones públicos en la zona Metropolitana del país, 2020", en el cual se mencionan las muertes y cremaciones por COVID-19 en todo México durante cierto periodo, y proporcionan el porcentaje de incremento de cremaciones el cual indica que durante 2020 aumentaron las cremaciones a un $76.8 \%{ }^{(16)}$.

Una vez obtenido el número de muertes en 2020 y un estimado del \% de cremaciones, se calculó que alrededor de 101,953 personas fueron cremadas, usando este dato para calcular el total de mg/cuerpo (tabla 5) de los contaminantes analizados por la normatividad mexicana.

Tabla 5. Resultados de contaminantes totales en mg/cuerpo, que sí son medidos por la norma NADF-017-AIRE-2017 (del año 2020).

\begin{tabular}{|c|c|c|}
\hline Contaminante & Factor de emisión (mg/cuerpo) & $\begin{array}{c}\text { Total, por cuerpos en 2020 } \\
\text { (mg/cuerpo) }\end{array}$ \\
\hline Partículas Suspendidas & $38,555.351$ & $3,930,824,755.662$ \\
\hline Monóxido de Carbono & $1.41 \times 10^{-7}$ & $1.44 \times 10^{-02}$ \\
\hline Óxidos de nitrógeno & $3.09 \times 10^{-7}$ & $3.15 \times 10^{-02}$ \\
\hline
\end{tabular}

Para el año 2021 es importante mencionar que la información proporcionada hasta el momento por el INEGI, no presenta puntualmente las muertes, por tanto, para hacer la estimación de estas, se involucran las defunciones esperadas por cualquier otra causa, información que fue proporcionada en la Página del Gobierno de México, en la sección de "Datos: Exceso de mortalidad" (18), y las muertes por COVID-19 proporcionadas por el Tecnológico de Monterrey ${ }^{(8)}$. Por lo tanto, para calcular las muertes totales en el semestre enero-junio de 2021, se consideró la estimación de las defunciones ya esperadas y se sumaron a las muertes por COVID-19.

Una vez obtenido el número de muertes durante ese periodo que fue alrededor de 75,499 personas y el estimado del \% de cremaciones considerado del 76.8\% (manteniendo el porcentaje del año 2020, debido a que aún no hay información solida de este), se calculó 
que alrededor de 57,983 personas fueron cremadas, usando este dato para calcular el total de mg/cuerpo (tabla 6) de los contaminantes analizados por la normatividad mexicana.

Tabla 6. Resultados de contaminantes totales en $\mathrm{mg} / \mathrm{cuerpo}$, que sí son medidos por la norma NADF-017-AIRE-2017 (del semestre enero-junio 2021).

\begin{tabular}{|c|c|c|}
\hline Contaminante & Factor de emisión (mg/cuerpo) & $\begin{array}{c}\text { Total, por cuerpos en primer } \\
\text { semestre 2021 (mg/cuerpo) }\end{array}$ \\
\hline Partículas Suspendidas & $38,555.351$ & $2,235,549,056.620$ \\
\hline Monóxido de Carbono & $1.41 \times 10^{-7}$ & $8.18 \times 10^{-03}$ \\
\hline Óxidos de nitrógeno & $3.09 \times 10^{-7}$ & $1.79 \times 10^{-02}$ \\
\hline
\end{tabular}

En la tabla resumen (tabla 7) se representan los totales en mg/cuerpo de contaminantes producidos y las variaciones para los años 2019-2020 y 2019-enero-junio 2021, finalmente se muestra el incremento total que hubo de los contaminantes durante este periodo de la pandemia.

Tabla 7. Resumen de incremento total en $\mathrm{kg}$ de los contaminantes mencionados en la normatividad mexicana de la CDMX.

\begin{tabular}{|c|c|c|c|c|c|c|c|}
\hline Contaminante & $\begin{array}{c}\text { Factor de } \\
\text { emisión } \\
\text { (mg/cuerpo) }\end{array}$ & $\begin{array}{c}\text { Total, por } \\
\text { cuerpos en } \\
2019 \\
\text { (mg/cuerpo) }\end{array}$ & $\begin{array}{c}\text { Total, por } \\
\text { cuerpos en } 2020 \\
\text { (mg/cuerpo) }\end{array}$ & $\begin{array}{c}\text { Total, por } \\
\text { cuerpos en 2021 } \\
\text { (mg/cuerpo) }\end{array}$ & $\begin{array}{l}\text { Variación } \\
2019-2020 \\
\text { (kg cont.) }\end{array}$ & $\begin{array}{l}\text { Variación } \\
2019-2021 \\
\text { (kg cont.) }\end{array}$ & $\begin{array}{l}\text { Incremento } \\
\text { total (kg } \\
\text { cont.) }\end{array}$ \\
\hline $\begin{array}{c}\text { Partículas } \\
\text { Suspendidas }\end{array}$ & $38,555.351$ & $1,294,349,477$ & $3,930,824,755.662$ & $2,235,549,056.620$ & $1,757,868.470$ & $62,592.770$ & $1,820,461.240$ \\
\hline $\begin{array}{l}\text { Monóxido de } \\
\text { Carbono }\end{array}$ & $1.41 \times 10^{-7}$ & $4.73 \times 10^{-03}$ & $1.44 \times 10^{-02}$ & $8.18 \times 10^{-03}$ & $6.43 \times 10^{-06}$ & $2.29 \times 10^{-07}$ & $6.66 \times 10^{-06}$ \\
\hline $\begin{array}{l}\text { Óxidos de } \\
\text { nitrógeno }\end{array}$ & $3.09 \times 10^{-7}$ & $1.04 \times 10^{-02}$ & $3.15 \times 10^{-02}$ & $1.79 \times 10^{-02}$ & $1.41 \times 10^{-05}$ & $5.02 \times 10^{-07}$ & $1.46 \times 10^{-05}$ \\
\hline
\end{tabular}

Nota: Cabe destacar que se consideraron sólo los parámetros medidos por la NADF017-AIRE-2017, que es la norma correspondiente para los equipos de cremación e incineración en la CDMX, (esta norma mide partículas suspendidas, monóxido de carbono y los óxidos de nitrógeno).

De los datos mostrados en las tablas 4, 5 y 6, se concluye que la cantidad de contaminantes, partículas suspendidas, monóxido de carbono, óxidos de nitrógeno, emitidos por cada cuerpo cremado en el año 2020, superó a las emisiones del año 2019. 
Una vez obtenidos estos resultados y "resaltando" una variación negativa para el ambiente, se indagaron diferentes notas en las que se menciona y confirma la generación de más gases contaminantes por los diferentes componentes de la cremación, notas como: En una petición realizada al Honorable Congreso del CDMX se indica que: "Se supone que estas emisiones se pueden controlar como las emisiones de un cuerpo sin COVID-19 con la Norma Oficial Mexicana NOM-043-SEMARNAT-1993, que establece los Límites Máximos Permisibles de emisiones a la atmósfera de partículas provenientes de fuentes fijas. Pero la norma está diseñada para establecimientos que cumplen con procesos complejos de operación del horno crematorio, lo que nos indica que las emisiones que son generadas por los hornos usados actualmente en México, bajo las circunstancias en las que nos encontramos al ser evaluadas con la misma norma, presentaran incongruencias y huecos normativos que no son suficientes para un control y cumplimiento adecuado", menciona el Diputado Sánchez ${ }^{(19)}$.

De forma no menos importante se encontró un reporte del año 2019, realizado por "Grupo Legislativo Morena”, en el cual se menciona casi lo mismo que en el informe del diputado Sánchez, pero a nombre de la Diputada Espinosa de los Monteros Julia:

"Hoy en día, los hornos crematorios, son evaluados de conformidad con la Norma Oficial Mexicana NOM-043-SEMARNAT-1993, donde se establecen los LMP de emisión a la atmósfera de partículas sólidas provenientes de fuentes fijas (en este caso, los hornos crematorios). Dicha norma fue diseñada para evaluar las emisiones a la atmósfera, originadas por la actividad de establecimientos industriales que cuenten con procesos más complejos comparados con los de un horno crematorio, por lo tanto, los hornos aún se regulan bajo esta norma y por consecuencia, dichos establecimientos deben cumplir con el LMP de los contaminantes que la misma señala, pero no podemos comparar las emisiones provenientes de procesos de índole industrial, con las emisiones que se generan de la combustión de los cadáveres y restos humanos."(20)

Como tal, haciendo una investigación más profunda, la teoría indica que en el proceso de la cremación de un cadáver no sólo se producen los tres contaminantes ya mencionados, sino que también se producen otros, ya sean emitidos por la combustión, por el féretro o por el cadáver. Cuando un cuerpo es cremado, usualmente libera entre 0.8 y 5.9 gramos de mercurio (liberado pasado 40 min. después de que inicia la cremación). El 75\% de este 
material suele dirigirse al aire, mientras que el $25 \%$ se dirige al suelo y agua; este contaminante genera daños en el sistema nervioso central de los humanos ${ }^{(21)}$.

Según la información que nos proporciona el Departamento de Ciencias de la Ingeniería Ambiental de la Universidad de Florida, los procesos emiten estos contaminantes por medio de 3 vías diferentes:

- Combustión: Esta es responsable de la emisión de partículas de cloruro de hidrógeno.

- Combustión incompleta: En este caso se produce el monóxido de carbono.

- Volatilización de metales preexistentes en el cadáver: es responsable de contaminantes tales como: mercurio, cadmio, plomo, monóxido de carbono, material particulado, cloruro de hidrógeno, óxido de nitrógeno, dióxido de sulfuro y dioxinas. (21)

Por tanto, para estos contaminantes, apoyándonos del factor de emisión ya antes mencionado, también se realizó el cálculo en mg/cuerpo, con la misma cantidad de muertes para los años 2019 (tabla 8), 2020 (tabla 9) y primer semestre del 2021 (tabla 10), y el mismo \% de cremaciones.

Tabla 8. Resultados de contaminantes no monitoreados ni analizados por la normatividad actual en la CDMX (totales durante el año 2019).

\begin{tabular}{|c|c|c|c|c|c|}
\hline Contaminantes & $\begin{array}{c}\text { Factor de } \\
\text { emisión } \\
\text { (mg/cuerpo) }\end{array}$ & $\begin{array}{c}\text { Total, por } \\
\text { cuerpos en } 2019 \\
(\mathrm{mg} / \text { cuerpo })\end{array}$ & Contaminantes & $\begin{array}{c}\text { Factor de } \\
\text { emisión } \\
\text { (mg/cuerpo) }\end{array}$ & $\begin{array}{c}\text { Total, por } \\
\text { cuerpos en } 2019 \\
\text { (mg/cuerpo) }\end{array}$ \\
\hline $\begin{array}{l}\text { Dióxido de Azufre } \\
\left(\mathrm{SO}_{2}\right)\end{array}$ & $5.44 \times 10^{-07}$ & $3.07 \times 10^{-02}$ & Antimonio (Sb) & 13.698 & 772,011 \\
\hline Plomo $(\mathrm{Pb})$ & 30.028 & $1,692,359.937$ & Bario $(\mathrm{Ba})$ & 13.608 & $766,938.660$ \\
\hline $\begin{array}{c}\text { Fluoruro de } \\
\text { Hidrogeno o Ácido } \\
\text { Fluorhídrico } \\
\text { cuando esta } \\
\text { hidratado (HF) }\end{array}$ & 297.103 & $16,744,545.570$ & Cobre $(\mathrm{Cu})$ & 12.428 & $700,434.570$ \\
\hline Arsénico (As) & 13.608 & $766,938.660$ & $\begin{array}{c}\text { Ácido Clorhídrico } \\
(\mathrm{HCl})\end{array}$ & 32658.651 & $1,840,621,838$ \\
\hline Cadmio (Cd) & 5.035 & 283,770 & Mercurio (Hg) & 1492.319 & $84,106,197.180$ \\
\hline Cromo $(\mathrm{Cr})$ & 6.123 & $345,088.580$ & $\begin{array}{l}\text { Hidrocarburos } \\
\text { Aromáticos } \\
\text { Policíclicos } \\
\text { (PAH's) }\end{array}$ & 0.013 & 732.672 \\
\hline Dioxinas y Furanos & 0.0000168 & 0.947 & I & I & I \\
\hline
\end{tabular}

Tabla 9. Resultados de contaminantes no monitoreados ni analizados por la normatividad actual en la CDMX (totales durante el año 2020). 
Estimación del incremento de las...

\begin{tabular}{|c|c|c|c|c|c|}
\hline Contaminantes & $\begin{array}{c}\text { Factor de } \\
\text { emisión } \\
\text { (mg/cuerpo) }\end{array}$ & $\begin{array}{c}\text { Total, por } \\
\text { cuerpos en } 2020 \\
\text { (mg/cuerpo) }\end{array}$ & Contaminantes & $\begin{array}{c}\text { Factor de } \\
\text { emisión } \\
\text { (mg/cuerpo) }\end{array}$ & $\begin{array}{l}\text { Total, por cuerpos } \\
\text { en } 2020(\mathrm{mg} / \text { cuerpo })\end{array}$ \\
\hline $\begin{array}{l}\text { Dióxido de Azufre } \\
\qquad\left(\mathrm{SO}_{2}\right)\end{array}$ & $5.44 \times 10^{-07}$ & $5.55 \times 10^{-02}$ & Antimonio (Sb) & 13.698 & $1,396,549.020$ \\
\hline Plomo $(\mathrm{Pb})$ & 30.028 & $3,061,437.718$ & Bario $(\mathrm{Ba})$ & 13.608 & $1,387,373.270$ \\
\hline $\begin{array}{c}\text { Fluoruro de } \\
\text { Hidrogeno o Ácido } \\
\text { Fluorhídrico cuando } \\
\text { esta hidratado (HF) }\end{array}$ & 297.103 & $30,290,473.230$ & Cobre $(\mathrm{Cu})$ & 12.428 & $1,267,069$ \\
\hline Arsénico (As) & 13.608 & $1,387,373.270$ & $\begin{array}{l}\text { Ácido Clorhídrico } \\
\text { (HCl) }\end{array}$ & 32658.651 & 3,329,639,868.600 \\
\hline Cadmio (Cd) & 5.035 & $513,332.190$ & Mercurio (Hg) & 1492.319 & $152,146,052.790$ \\
\hline Cromo $(\mathrm{Cr})$ & 6.123 & $624,256.800$ & PAH's & 0.013 & $1,325.386$ \\
\hline Dioxinas y Furanos & 0.0000168 & 1.713 & l & l & l \\
\hline
\end{tabular}

Tabla 10. Resultados de contaminantes no monitoreados ni analizados por la normatividad actual en la CDMX (totales durante el semestre enero-junio 2021).

\begin{tabular}{|c|c|c|c|c|c|}
\hline Contaminantes & $\begin{array}{c}\text { Factor de } \\
\text { emisión } \\
\text { (mg/cuerpo) }\end{array}$ & $\begin{array}{c}\text { Total, por } \\
\text { cuerpos en } 2021 \\
\text { (mg/cuerpo) }\end{array}$ & Contaminantes & $\begin{array}{c}\text { Factor de } \\
\text { emisión } \\
\text { (mg/cuerpo) }\end{array}$ & $\begin{array}{l}\text { Total, por cuerpos en } \\
2021 \text { (mg/cuerpo) }\end{array}$ \\
\hline $\begin{array}{l}\text { Dióxido de Azufre } \\
\qquad\left(\mathrm{SO}_{2}\right)\end{array}$ & $5.44 \times 10^{-07}$ & $3.1510^{-02}$ & Antimonio (Sb) & 13.698 & $794,249.050$ \\
\hline Plomo $(\mathrm{Pb})$ & 30.028 & $1,741,108.960$ & Bario (Ba) & 13.608 & $789,030.600$ \\
\hline $\begin{array}{c}\text { Fluoruro de } \\
\text { Hidrogeno o } \\
\text { Ácido } \\
\text { Fluorhídrico } \\
\text { cuando esta } \\
\text { hidratado (HF) }\end{array}$ & 297.103 & $17,226,878.090$ & Cobre $(\mathrm{Cu})$ & 12.428 & $720,610.830$ \\
\hline Arsénico (As) & 13.608 & $789,030.600$ & $\begin{array}{l}\text { Ácido Clorhídrico } \\
(\mathrm{HCl})\end{array}$ & 32658.651 & $1,893,641,596.818$ \\
\hline Cadmio $(\mathrm{Cd})$ & 5.035 & $291,943.640$ & Mercurio (Hg) & 1492.319 & $86,528,905.740$ \\
\hline Cromo $(\mathrm{Cr})$ & 6.123 & $355,028.980$ & PAH's & 0.013 & 753.777 \\
\hline $\begin{array}{l}\text { Dioxinas y } \\
\text { Furanos }\end{array}$ & 0.0000168 & 0.974 & I & I & I \\
\hline
\end{tabular}

Tabla 11. Resumen de contaminantes no monitoreados ni analizados por la normatividad actual en la CDMX. 


\begin{tabular}{|c|c|c|c|c|c|c|c|}
\hline Contaminante & $\begin{array}{c}\text { Factor de } \\
\text { emisión } \\
\text { (mg/cuerpo) }\end{array}$ & $\begin{array}{c}\text { Total, por } \\
\text { cuerpos en } \\
2019 \\
\text { (mg/cuerpo) }\end{array}$ & $\begin{array}{c}\text { Total, por } \\
\text { cuerpos en } \\
2020 \\
\text { (mg/cuerpo) }\end{array}$ & $\begin{array}{c}\text { Total, por } \\
\text { cuerpos en } \\
2021 \\
\text { (mg/cuerpo) }\end{array}$ & $\begin{array}{l}\text { Variación } \\
2019-2020 \\
\text { (kg cont.) }\end{array}$ & $\begin{array}{l}\text { Variación } \\
2019- \\
2021 \text { (kg } \\
\text { cont.) }\end{array}$ & $\begin{array}{c}\text { Incremento } \\
\text { total }\end{array}$ \\
\hline $\mathrm{SO}_{2}$ & $5.44 \times 10^{-07}$ & $3.07 \times 10^{-02}$ & $5.55 \times 10^{-02}$ & $3.15 \times 10^{-02}$ & $2.48 \times 10^{-05}$ & $8.83 \times 10^{-07}$ & $2.57 \times 10^{-05}$ \\
\hline Plomo & 30.028 & $1,692,359.937$ & $3,061,437.718$ & $1,741,108.960$ & $1,369.080$ & 48.750 & $1,417.830$ \\
\hline $\mathrm{HF}$ & 297.103 & $16,744,545.570$ & $30,290,473.230$ & $17,226,878.090$ & $13,545.930$ & 482.330 & $14,028.260$ \\
\hline As & 13.608 & $766,938.660$ & $1,387,373.270$ & $789,030.600$ & 620.430 & 22.090 & 642.530 \\
\hline $\mathrm{Cd}$ & 5.035 & 283,770 & $513,332.190$ & $291,943.640$ & 229.560 & 8.170 & 237.740 \\
\hline $\mathrm{Cr}$ & 6.123 & $345,088.580$ & $624,256.800$ & $355,028.980$ & 279.170 & 9.940 & 289.110 \\
\hline $\begin{array}{l}\text { dioxinas y } \\
\text { furanos** }\end{array}$ & 0.0000168 & 0.947 & 1.713 & 0.974 & 0.766 & 0.0270 & 0.793 \\
\hline $\mathrm{Sb}$ & 13.698 & 772,011 & $867,368.760$ & $794,249.050$ & 624.540 & 22.240 & 646.780 \\
\hline $\mathrm{Ba}$ & 13.608 & $766,938.660$ & $861,669.880$ & $789,030.600$ & 620.430 & 22.090 & 642.530 \\
\hline $\mathrm{Cu}$ & 12.428 & $700,434.570$ & $786,951.300$ & $720,610.830$ & 566.630 & 20.180 & 586.810 \\
\hline $\mathrm{HCl}$ & 32658.651 & $1,840,621,838$ & $2,067,972,953.320$ & $1,893,641,596.818$ & $1,489,018.030$ & $53,019.760$ & $1,542,037.790$ \\
\hline $\mathrm{Hg}$ & 1492.319 & $84,106,197.180$ & $94,494,880.690$ & $86,528,905.740$ & $68,039.860$ & $2,422.710$ & $70,462.560$ \\
\hline PAH's & 0.013 & 732.672 & 823.171 & 753.777 & 0.590 & 0.020 & 0.610 \\
\hline
\end{tabular}

**Nota: El incremento de todos los contaminantes mencionados en la tabla 11, maneja las unidades en $\mathrm{kg}$ de contaminante, a excepción de las dioxinas y furanos que son contaminantes reportados por la norma NOM-098-SEMARNAT-2002 por las unidades EQT que refieren a $\mathrm{mg} / \mathrm{m}^{3}$, así que se reporta el incremento en unidades de $\mathrm{mg}$.

En las tablas 8, 9 y 10, se observa que, tal como sucede con los contaminantes Partículas Suspendidas, Monóxido de Carbono y Óxidos de Nitrógeno (aun si estos dos últimos tuvieron un incremento no significativo), los demás contaminantes $\mathrm{SO}_{2}, \mathrm{~Pb}, \mathrm{HF}, \mathrm{As}, \mathrm{Cd}$, $\mathrm{Cr}, \mathrm{Sb}, \mathrm{Ba}, \mathrm{Cu}, \mathrm{NOx}, \mathrm{CO}$, así como PAH's, superaron los valores del año 2019, al igual que los contaminantes dioxinas y furanos, $\mathrm{HCl}$ y $\mathrm{Hg}$ en especial. Todo lo anterior se relaciona fundamentalmente, con un proceso de cremación que no cumplió con las Buenas Prácticas de Combustión.

Por ejemplo, personas que trabajan en una funeraria de México dijeron: "meten hasta dos cuerpos en un servicio por la demanda" (22). Esta es una violación que puede provocar el exceso de emisión de contaminantes durante la cremación, o sea, la quema simultanea de dos o más cuerpos, lo cual se considera una práctica ilegal ${ }^{(23)}$. Por otra parte, los accesorios del cadáver, ya sea joyería como relojes, anillos, pendientes, pulseras, etc. son 
removidos del cuerpo y entregados a la familia. En caso de que el difunto ocupara marcapasos este deberá ser removido debido a que puede explotar y dañar el horno.

Una vez identificados los contaminantes y habiendo obtenido su incremento, se consideró importante conocer los diferentes efectos que tienen para la salud y el medio ambiente, reconociendo con esto, la problemática que representa la cremación de cadáveres sin un control eficiente de emisiones contaminantes.

Tabla 12. Efecto de los 3 contaminantes mas dañinos para el medio ambiente y salud.

\begin{tabular}{|c|c|c|}
\hline Contaminante & Efecto Ambiental & Efecto en la Salud \\
\hline $\begin{array}{c}\text { Dioxinas y } \\
\text { Furanos [mg] }\end{array}$ & $\begin{array}{l}\text { Permanecen en el aire, agua o suelo por cientos de } \\
\text { años, debido a que son estables y tienen la capacidad } \\
\text { de resistir los procesos de degradación tanto físicos } \\
\text { como químicos. }\end{array}$ & $\begin{array}{l}\text { Las dioxinas tienen elevada toxicidad y pueden provocar } \\
\text { problemas de reproducción y desarrollo, afectar el sistema } \\
\text { inmunitario, interferir con hormonas y, de ese modo, } \\
\text { causar cáncer. } \\
\text { No existen en la naturaleza, por lo tanto, los seres vivos } \\
\text { no tienen la capacidad de metabolizarlos o detoxificarlos, } \\
\text { haciéndolos así también resistentes a la degradación } \\
\text { biológica. }\end{array}$ \\
\hline $\begin{array}{c}\text { Cloruro de } \\
\text { Hidrogeno }[\mathrm{kg}]\end{array}$ & $\begin{array}{l}\text { Cuando este contaminante es liberado a la atmosfera } \\
\text { puede ser removido por la lluvia, este se disocia } \\
\text { fácilmente con el agua debido a los iones de cloruro } \\
\text { e hidrogeno, lo cual puede reducir el pH del agua y } \\
\text { acidificarla. }\end{array}$ & $\begin{array}{l}\text { Este compuesto es irritante y corrosivo para cualquier } \\
\text { tejido con el que tiene contacto. } \\
\text { La exposición breve a niveles altos puede producir } \\
\text { respiración jadeante, estrechamiento de los bronquiolos, } \\
\text { coloración azul de la piel, acumulación de líquido en los } \\
\text { pulmones y aun la muerte. } \\
\text { La exposición a niveles aún más altos puede producir } \\
\text { hinchazón y espasmos de la garganta, así como asfixia. }\end{array}$ \\
\hline $\begin{array}{c}\text { Partículas } \\
\text { Suspendidas } \\
{[\mathrm{kg}]}\end{array}$ & $\begin{array}{l}\text { Al poder viajar largas distancias por medio del aire, } \\
\text { las Partículas suspendidas son la causa de: } \\
\text { 1. Que lagos y arroyos se vuelvan ácidos. } \\
\text { 2. Cambios en el balance nutricional de grandes } \\
\text { cuencas fluviales. } \\
\text { 3. Reducción de los nutrientes del suelo. } \\
\text { 4. Daño en los bosques sensibles y cultivos } \\
\text { agrícolas. } \\
\text { 5. Efectos perjudiciales sobre la diversidad de } \\
\text { ecosistemas. }\end{array}$ & $\begin{array}{l}\text { Las partículas más dañinas para la salud son las de } 10 \\
\text { micrómetros de diámetro, o menos ( } \leq \text { PM10), que pueden } \\
\text { penetrar y alojarse en el interior profundo de los } \\
\text { pulmones. La exposición crónica a las partículas agrava el } \\
\text { riesgo de desarrollar cardiopatías y neumopatías, así como } \\
\text { cáncer de pulmón. }\end{array}$ \\
\hline
\end{tabular}

\section{CONCLUSIÓN O CONSIDERACIONES FINALES}

De acuerdo con la investigación realizada podemos concluir que el incremento de muertes por COVID-19 y el correspondiente aumento en las cremaciones de cadáveres, aumentó 
de manera considerable los niveles de emisiones de gases contaminantes a la atmósfera en la CDMX. Situación que es contrastante con lo vivido en otras ciudades del mundo ya que en la mayoría de ellas la calidad del aire mejoró; ahora podemos asegurar que el deficiente proceso de cremación que se lleva a cabo en la CDMX está relacionado directamente a la mala calidad del aire, y esto se ve reflejado en los resultados obtenidos en la investigación.

Al utilizar los factores de emisión, se pudo obtener el incremento de las emisiones contaminantes a la atmósfera producto de la cremación de cadáveres por COVID-19, que representa una variación del $72.9 \%$ (antes de la pandemia), a un $76.8 \%$ a diciembre del 2020, cabe destacar que entre 2019 y junio de 2021 hubo un incremento que representó cremar adicionalmente alrededor de 47,217 cuerpos.

Una situación preocupante es haber identificado que en la NADF-017-AIRE-2017 sólo se están monitoreando y analizando 3 contaminantes, situación que contrasta con la normatividad de la USEPA, Unión Europea y de Colombia; que muestrea y analiza Ácido Clorhídrico, Dioxinas y Furanos, Mercurio, entre otros contaminantes.

Los resultados de la cuantificación de emisiones a la atmósfera que no están siendo muestreadas ni analizadas por la normatividad actual, representan un alto riesgo de impacto a la salud de la población y al ambiente. Más de 1,542,037.790 kg de Ácido clorhídrico, aunado a más de 1,417.830 kg de plomo con más de 70,462.560 kg mercurio y $0.793 \mathrm{mg}$ de dioxinas y furanos, son de las cantidades adicionales de contaminantes que se han emitido a la atmósfera en el periodo de estudio durante la pandemia, y que impactan y siguen impactando de manera importante a la ya contaminada atmósfera de la CDMX.

Lo más grave para la salud de la población y para el ambiente, es que todos estos contaminantes seguirán emitiéndose a la atmósfera, suelo y agua de la CDMX; aun después de que termine la pandemia, por lo que es urgente tomar en cuenta los resultados de este proyecto para aplicar acciones correctivas a la brevedad posible, y continuar con las investigaciones necesarias.

\section{RECOMENDACIONES}


- Actualización inmediata de la norma NADF-017-AIRE-2017 que incluya más Límites Máximos Permisibles de contaminantes producto de la cremación de cadáveres (Así lo hace Colombia, USA y gran parte de la Comunidad Europea).

- Crear una nueva Norma Oficial Mexicana que regule toda la cremación de cadáveres en México.

- Utilizar la palabra Cremación con una definición sólo para cadáveres y el término Incineración para residuos.

- Debido a que los equipos de incineración de residuos sí cuentan con tecnologías para el control de las emisiones, sería ideal considerarlos para utilizarlos en el proceso de cremación, ya que la presencia de elementos distintos al C, H y O, como pueden ser $\mathrm{N}, \mathrm{S}, \mathrm{P}, \mathrm{Cl}$ y otros halógenos, conduce a la formación de productos como $\mathrm{NOx}, \mathrm{SO}_{2}$, $\mathrm{HCl}$ y otros, derivados de algunos de ellos por reacciones secundarias, como clorodibenzodioxinas y clorodibenzofuranos, que contaminan los gases de combustión y exigen el adecuado tratamiento de los mismos antes de su emisión a la atmósfera.

- Realizar protocolos de pruebas preoperativas a cualquier equipo de Cremación que deba autorizarse, incluyendo nuevos y en operación.

- Realizar visitas de inspección frecuentes a Crematorios autorizados con la finalidad de comprobar la correcta operación y el cumplimiento de la normatividad.

\section{Definiciones y Terminología}

III.1 Inhumación: Enterrar un cadáver.

III.2 Incineración: Según la norma NADF-017-AIRE-2017, es el proceso mediante el cual los cadáveres de animales y sus restos se someten a altas temperaturas controladas con el fin de reducirlos a cenizas ${ }^{(1)}$.

III.3 Cremación: Según la norma NADF-017-AIRE-2017 se indica que, es el proceso mediante el cual un cadáver, restos humanos o restos humanos áridos se someten a altas temperaturas controladas con el fin de reducirlos a cenizas ${ }^{(1)}$, (Si no se realiza de acuerdo como la normatividad lo indica puede generar dioxinas y furanos).

III.4 Agente Biológico Infeccioso: según la norma NOM-087-ECOL-SSA1-2002, es cualquier microorganismo capaz de producir enfermedades cuando está presente en concentraciones suficientes (inóculo), en un ambiente propicio (supervivencia), en un hospedero susceptible y en presencia de una vía de entrada. ${ }^{(23)}$ 
III.5 Residuos Peligrosos Biológico-Infecciosos: Son aquellos materiales generados durante los servicios de atención médica que contengan agentes biológico-infecciosos según son definidos en la norma NOM-087-ECOL-SSA1-2002, y que puedan causar efectos nocivos a la salud y al ambiente ${ }^{(23)}$. (ver capítulo de Juan)

III.6 Emisión: Según la NOM-098-SEMARNAT-2001, es la descarga a la atmósfera de toda sustancia en cualquiera de sus estados físicos o de energía ${ }^{(2)}$.

III.7 Combustión: De acuerdo con lo que menciona la norma NOM-098-SEMARNAT2002, es el proceso controlado de oxidación rápida, que sucede durante la combinación de oxígeno con aquellos materiales o sustancias contenidas en los residuos con capacidad de oxidarse ${ }^{(2)}$.

III.8 Incinerador: Según la norma NOM-098-SEMARNAT-2001, es el equipo empleado para la oxidación térmica de residuos, con o sin recuperación de calor producido por la combustión, con sus respectivos dispositivos de control de temperatura y de composición de gases, así como con tolvas para la recepción de cenizas ${ }^{(2)}$.

III.9. Fuente fija: son los establecimientos mercantiles y de servicio que emiten contaminantes al ambiente, ubicados en el territorio de la Ciudad de México.

\section{LISTA DE REFERENCIAS}

Secretaria del Medio Ambiente de la CDMX. (2018). Norma Ambiental para el Distrito Federal, NADF-017-AIRE-2017. Equipos de cremación e incineración-Límites Máximos Permisibles a la atmósfera y condiciones de operación, Gobierno de la Ciudad de

México. http://data.sedema.cdmx.gob.mx/padla/index.php?option=com_content\&view=a rticle\&id=333:nadf017aire2017\&catid=53:normas-ambientales-del-distritofederal\&Itemid $=87$

Secretaria del Medio Ambiente y Recursos Naturales. (2004). Norma Oficial Mexicana, NOM-098-SEMARNAT-2002, Protección ambiental- Incineración de residuos, especificaciones de operación y límites de emisión de contaminantes, D.O.Fhttps://www.profepa.gob.mx/innovaportal/file/1309/1/nom-098-semarnat2002.pdf

Cárdenas, M.A. (2018, octubre). Gaceta del Senado. Iniciativa con proyecto de decreto por el que se reforman y adicionan diversas disposiciones de la ley general de salud en materia de incineración e inhumación de restos humanos y animales. 
México,

CDMX.

Recuperado

de:

https://www.senado.gob.mx/64/gaceta_del_senado/documento/84158

Organización Panamericana de la Salud. (2014, marzo). OMS estima que 7 millones de muertes ocurren cada año debido a la contaminación atmosférica. Recuperado dehttps://www3.paho.org/hq/index.php?option=com_content\&view=article\&id= 9406:2014-7-million-deaths-annually-linked-air-

pollution\&Itemid=135\&lang=es

Reforman de los artículos 314 y 348 de la Ley General de la Salud, en materia de disposición final de cadáveres, de 11 de febrero, Cámara de Diputados, iniciativa de

reforma

dehttp://sil.gobernacion.gob.mx/Archivos/Documentos/2020/02/asun_4001410_ 20200218_1581445951.pdf

El universal. (2020, mayo). ¿Cómo nos afecta la contaminación por hornos crematorios?

Dehttps://www.eluniversal.com.co/salud/como-nos-afecta-la-contaminacionpor-hornos-crematorios-DD2849995

V. Suárez, M. Suarez Quezada, S. Oros Ruiz, y E. Ronquillo De Jesús. (2020). Epidemiologia de COVID-19, del 19 en México del 27 de febrero al 30 de abril

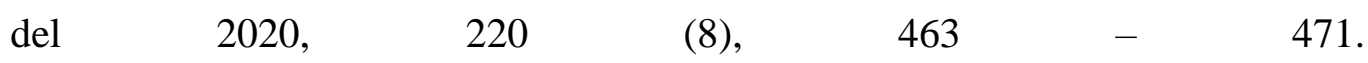
https://www.ncbi.nlm.nih.gov/pmc/articles/PMC7250750/

Tecnológico de Monterrey. (2021). COVID-19 en México. Recuperado dehttps://www.mexicovid19.app/covid-19

Secretaria de Salud. (2020). Guía de Manejo de Cadáveres por COVID-19 (Sars-CoV-2) en México. https://coronavirus.gob.mx/wpcontent/uploads/2020/04/Guia_Manejo_Cadaveres_COVID-19.pdf

El Sol de México. (2020, noviembre). Revisaran que crematorio de San Isidro cumpla normatividad tras denuncias. https://www.elsoldemexico.com.mx/metropoli/cdmx/revisaran-que-crematoriode-san-isidro-cumpla-normatividad-tras-denuncias-6039292.html

United States Environmental Protection Agency (1992), Emissions Testing of a Propane Fired Incinerator at a Crematorium. (Confidential Report No. ERC-39) http://cfpub.epa.gov/oarweb/index.cfm. 
Canberra Cementeries. (2009). Air Quality Impact Assessment Proposed Crematorium Tuggeranong,

$(\mathrm{ACT})$ https://www.cityservices.act.gov.au/_data/assets/pdf_file/0003/395400/302443R1R0.pdf

Funeral Natural a sustainable death. (sin fecha). La cremación y la Calidad del Aire. https://www.funeralnatural.net/articulos/la-cremacion-y-la-calidad-del-aire

Instituto Nacional de Estadística y Geografía. (2019). Características de las defunciones registradas en México durante 2019. Recuperado de https://www.inegi.org.mx/contenidos/saladeprensa/boletines/2020/EstSociodem o/DefuncionesRegistradas2019.pdf

Ramírez, M. [J García López]. (2017, 27 de marzo). C6 ¿Inhumación o cremación? [video].YouTube. https://www.youtube.com/watch?v=HqMgJfzsChM

Instituto Nacional de Estadística y Geografía. (2020). Recopilación de información de panteones públicos en zonas metropolitanas del país, 2020. México Recuperado dehttps://www.inegi.org.mx/contenidos/programas/ricpzmp/2020/doc/resultados _ricpzmp.pdf

Instituto Nacional de Estadística y Geografía. (2021). Características de las defunciones registradas en México durante 2020. Recuperado de https://www.inegi.org.mx/contenidos/saladeprensa/boletines/2021/EstSociode mo/DefuncionesRegistradas2020preliminar.pdf

Instituto Nacional de Salud Pública (INSP). (sin fecha). "Exceso de mortalidad por todas las causas durante la emergencia por covid 19, Mexico, 2020-2021.” Recuperado de https://coronavirus.gob.mx/exceso-de-mortalidad-en-mexico/

Sánchez, N. Consulta Congreso de la Ciudad de México, (2020). Recuperado de https://consulta.congresocdmx.gob.mx/consulta/webroot/img/files/iniciativa/IN 429_24_03112020.pdf

Grupo Legislativo Morena. Nuevo León (2019). Presenta Diputada Julia Espinosa de los Monteros Iniciativa de Ley con proyecto de decreto para regular la emisión a la atmósfera de partículas de los hornos crematorios. Recuperado de: http://www.hcnl.gob.mx/glmorena/2019/02/presenta-diputada-juliaespinosa-de-los-monteros-iniciativa-de-ley-con-proyecto-de-decreto-pararegu.php 
Cárdenas, M.A. México, CDMX. (2018) Iniciativa con proyecto de decreto por el que se reforman y adicionan diversas disposiciones de la ley general de salud en materia de incineración e inhumación de restos humanos y animales. Recuperado de: https://www.senado.gob.mx/64/gaceta_del_senado/documento/84158

Ayala, K. (2021). Vecinos de crematorio preparan queja por expedición de gases. El Sol de Zamora. Recuperado dehttps://www.elsoldezamora.com.mx/local/vecinos-decrematorio-preparan-queja-por-expedicion-de-gases-6325318.htm

Recondo, R.F. (2013). La Arquitectura del Crematorio. Función, estética y medio ambiente. $\quad$ EMPAI. Matanzas, Cuba. Recuperado dehttps://www.redalyc.org/pdf/1939/193929227002.pdf

Secretaria del Medio Ambiente y Recursos Naturales. (2003) Norma Oficial Mexicana, NOM-087-ECOL-SSA1-2002. Protección Ambiental-Salud Ambiental-Residuos Peligrosos Biológicos-Infecciosos-Clasificación y especificaciones de manejo, D.O.F. 17 de febrero de 2003. http://www.salud.gob.mx/unidades/cdi/nom/087ecolssa.html 